\title{
Particle position prediction based on Lagrangian coherency for flow over a cylinder in 4D-PTV
}

\author{
Ali Rahimi Khojasteh ${ }^{1 *}$, Dominique Heitz ${ }^{1}$, Yin Yang $^{1}$, Lionel Fiabane $^{1}$ \\ ${ }^{1}$ INRAE, OPAALE, F-35044, Rennes, France \\ * correspondent author: ali.rahimi-khojasteh@inrae.fr
}

\begin{abstract}
Recent developments in time-resolved Particle Tracking Velocimetry (4D-PTV) consistently improved tracking accuracy and robustness. We propose a novel technique named "Lagrangian coherent predictor" to estimate particle positions within the 4D-PTV algorithm. We add spatial and temporal coherency information of neighbour particles to predict a single trajectory using Lagrangian Coherent Structures (LCS). We found that even a weak signal from coherent neighbour motions improves particle prediction accuracy in complex flow regions. We applied Finite Time Lyapunov Exponent (FTLE) to quantify local boundaries (i.e. ridges) of coherent motions. Synthetic analysis of the wake behind a smooth cylinder at Reynolds number equal to 3900 showed enhanced estimation compared with the recent predictor functions employed in 4D-PTV. Results of the experimental study of the same flow configuration are reported. We compared predicted positions with the optimised final positions of Shake The Box (STB). It was found that the Lagrangian coherent predictor succeeded in estimating particle positions with minimum deviation to the optimised positions.
\end{abstract}

\section{Introduction}

This paper discusses a novel approach in time-resolved Particle Tracking Velocimetry (4D-PTV) to predict particle positions over space and time based on Lagrangian Coherent Structures (LCS). In the classic 4DPTV algorithm, predicted particle positions are given to the optimisation process for further corrections. The optimisation can deal with slight deviations between the predicted position and the true position. However, the optimisation fails to find the true position if the deviation is large enough to have multi-candidates for a single particle at $t_{n+1}$. This shows the importance of having an appropriate prediction in dense and complex motions. The proposed idea is initiated by arguing that predictions in PTV techniques focus on a single particle individually, while this single particle is not acting alone [1]. We propose this approach to locally differentiate coherent and non-coherent motions of neighbour particles around a single particle position to improve prediction accuracy, as shown in Figure 1. On the other hand, the information of coherent particles should be shared with each neighbour particle to predict their behaviour accurately and avoid misprediction. The present study was designed as a complementary function for 4D-PTV algorithms such as STB [2] and KLPT [3].

Briefly, it is assumed that particle positions are known for $n$ time steps (four to five). Afterwards, a mathematical prediction function is implemented to estimate particle positions for time-step $n+1$ followed by "shaking" and position refinement. It should be noted that the "shaking" process tries to look for a candidate true position very close to the predicted position. If misprediction happens, no matter how many times we perform "shaking", the true position is not achievable. This implies the importance of producing accurate predictions. This paper seeks to investigate the possibilities of improvements in motion estimation by adding meaningful physics into the prediction function. A simple prediction approach is a Polynomial function, suggested by Schanz et al. [4], resulting in reasonable predictions and 3D particle position reconstruction in simple flows [4, 5, 6]. However, significant off prediction occurs in case of flow associated with complexities such as high turbulence level, high Reynolds number, and mixing flows [7]. In such conditions, even by increasing the order of the Polynomial predictor functions from 3 to 10 [7], off prediction stays remained. The solution for this challenge is implementing optimal temporal filtering such as the Wiener filter, which has been first examined in 4D-PTV experiments by Schröder et al. [5]. Since then, this concept became consistent in the STB studies due to its high robustness and accurate motion estimations [8, 7]. As 


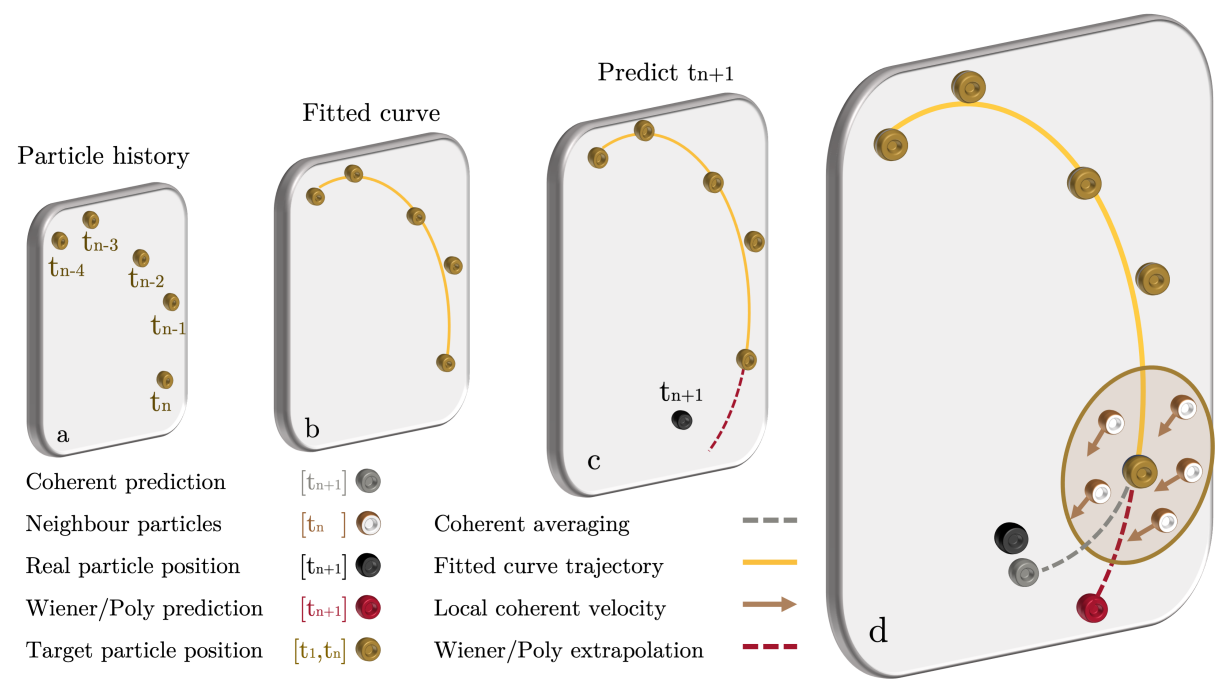

Figure 1: Particle prediction scenario from $t_{n}$ to $t_{n+1}:$ a), Known particle positions from history starting from $t_{n-4}$ up to $t_{n}$ (particle size is increasing gradually to show time-step differences); b), The trajectory (golden line) obtained from filtered curve fitting of known particle positions; c), Prediction based on extrapolating of the fitted trajectory (red dash line) from $t_{n}$ to $t_{n+1}$; d), Modified prediction (grey dash line) using velocity and acceleration information of coherent particles in neighbourhood of the target particle at $t_{n}$.

mentioned, the Wiener filter showed robust behaviour in prediction with complex flows but still suffers in high motion gradients. This implies the fact that the prediction function suffers from a lack of information to find true positions. Worth mentioning that these prediction-based techniques rely on one particle individually, excluding it from surroundings. All the information we know from an individual particle is its history. Even if we implement filtering and smoothing schemes such as STB using Wiener filter [8], our information is limited by the history of the target particle, ignoring that every particle is spatially and temporally coherent with a specific group of other particles following the same behaviour. This motivated us to take into account a group of coherent motions for predicting a single particle. We propose to locally determine information of coherent and non-coherent particles during the trajectory procedure by using the Finite-Time Lyapunov Exponent (FTLE). More details of coherent motion detection are discussed in Section 2 . After that, we address the prediction function with the minimisation approach in Section 3 . In the following Sections 4 and 5. we study and evaluate our proposed technique using synthetic and experimental case studies of the wake over and behind a smooth cylinder at Reynolds number equal to 3900.

\section{Coherent and non-coherent neighbours}

Every single particle is spatially and temporally coherent with a specific cluster of other particles following the same behaviour [1]. Figure 2 shows a schematic of coherent and non-coherent groups of neighbour particles in different colours evolving by time $\left(t_{1}-t_{5}\right)$. A particle can spatially meet a group of other particles in which there is no coherency link between them. There are many available concepts to identify Lagrangian Coherent Structures (LCS) from looking for separatrix lines or surfaces which divide structures into different coherent regions [9]. We introduced such a concept for the track initialisation in 4D-PTV studies, named Lagrangian Coherent Track Initialisation (LCTI), in synthetic flow configurations of the recent LPT Challenge [10] and for a real jet impingement experiment [1]. LCTI showed that FTLE is locally applicable for surrounding sparse trajectories to determine coherent neighbours.

To this end, we defined a local Eulerian frame around each particle, while all neighbourhood particles inside this area must be classified as coherent or non-coherent with the target particle. This frame is fixed during a series of time-steps that can provide an Eulerian view of the neighbourhood behaviour. Velocity values of the target particle are used to quantify the Eulerian frame size in each direction. If 2D / 3D velocity values are equal in each direction, then the shape would be a circle / sphere around the target particle; however, more directional gradients would adjust the shape (see Figure 1.d). All particles inside the Eulerian frame in the same phase or with phase delay are considered neighbourhoods. By tracking each 


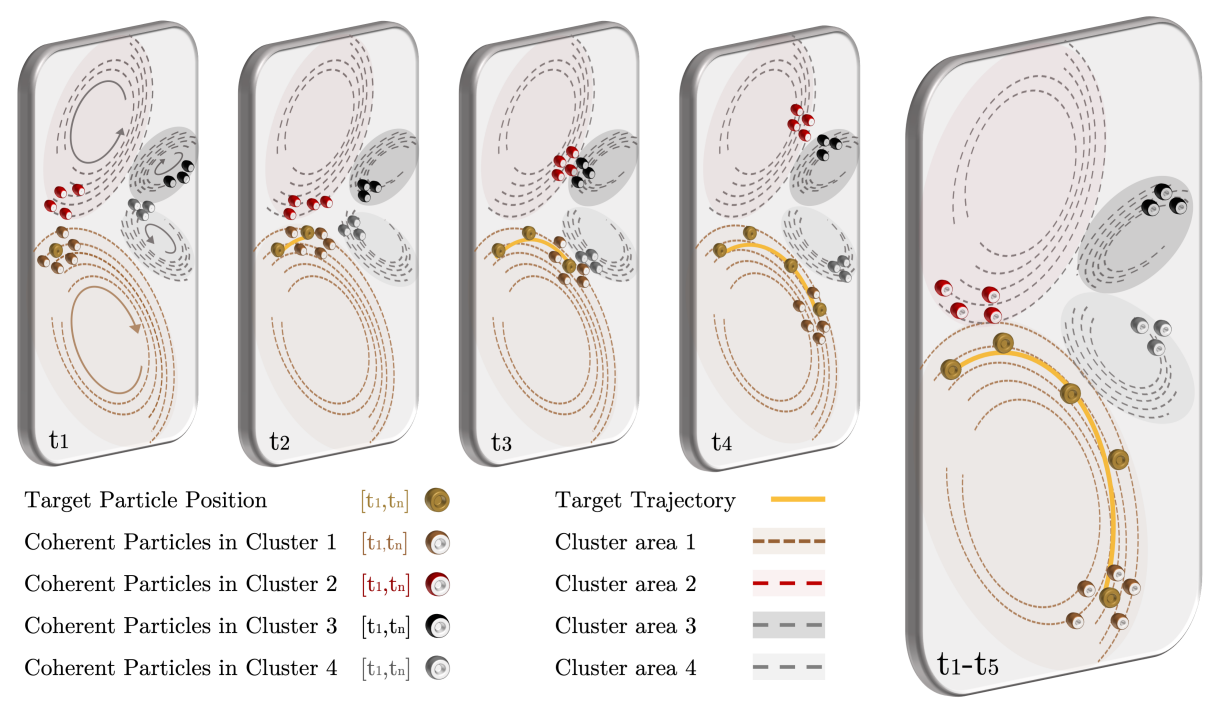

Figure 2: Schematic of particle trajectories in 2D pair vortices starting from $t_{1}$ to $t_{5}: t_{1}$ ), The target particle with coherent neighbour particles located in a clockwise vortex (golden cluster), non-coherent particles belong to different clusters; $t_{2}$ ), The target particle trajectory (golden line) approaching to particles in the red cluster; $t_{3}$ ), The target particle separation with non-coherent particles in the red cluster and approaching to particles in the grey cluster; $t_{4}$ ), Separation of non-coherent particles in the grey cluster with the target particle; $t_{1}-t_{5}$ ), Full trajectory view of the target particle alongside with coherent particles in golden cluster.

Lagrangian particle in the flow over a finite time (see Figure 2), we can compute the FTLE value based on neighbour trajectories as,

$$
\sigma_{t_{0}}^{t}=\frac{1}{|T|} \sqrt{\lambda_{\max }(\Delta)}=\frac{1}{|T|} \log \left(\frac{\delta x(t)}{\delta x\left(t_{0}\right)}\right)
$$

where $\sigma_{t_{0}}^{t}$ is named the scalar FTLE value showing the amount of stretching over the interval time $T=t-t_{0}$, and $\delta x$ is the displacement of neighbour particles. $\lambda_{\max }$ is the maximum eigenvalue of the right Cauchy-Green deformation tensor [11] obtained from the displacement map. A lower FTLE value means a neighbouring particle is coherent and acts in the same behaviour with the target particle spatially over a specific temporal scale. As a result of this classification, the mean values, including velocity and acceleration of the coherent neighbour particles, can be superimposed (weighted averaged) on the target particle (see Figure 11. This physics-based information is added to the resulting prediction function. The prediction function of this study is a 3rd order polynomial predictor that must satisfy particle history and additional coherent dynamics. Details of the prediction function is addressed in Section 3.

\section{Prediction Function}

Polynomial function is the most simple predictor that can be used in the time resolved particle tracking techniques. The polynomial coefficients must be determined optimally by minimising mean square error such that the corresponding polynomial curve with order of $n$ best fits the given positions. This can be formalised as,

$$
\sum_{j=n-m}^{n} \sum_{i=1}^{n+1} a_{i} \cdot t_{j}^{i-1}=y_{n-m, n}
$$

where $a_{i}$ is unknown coefficients of the predictor function. $y_{n-m, n}$ is known positions of the last finite frames (in this study $m=4$ ). Therefore, the least square cost function is,

$$
J=\frac{1}{n} \sum_{i=n-m}^{n}\left(X_{i}-y_{i}\right)^{2}
$$




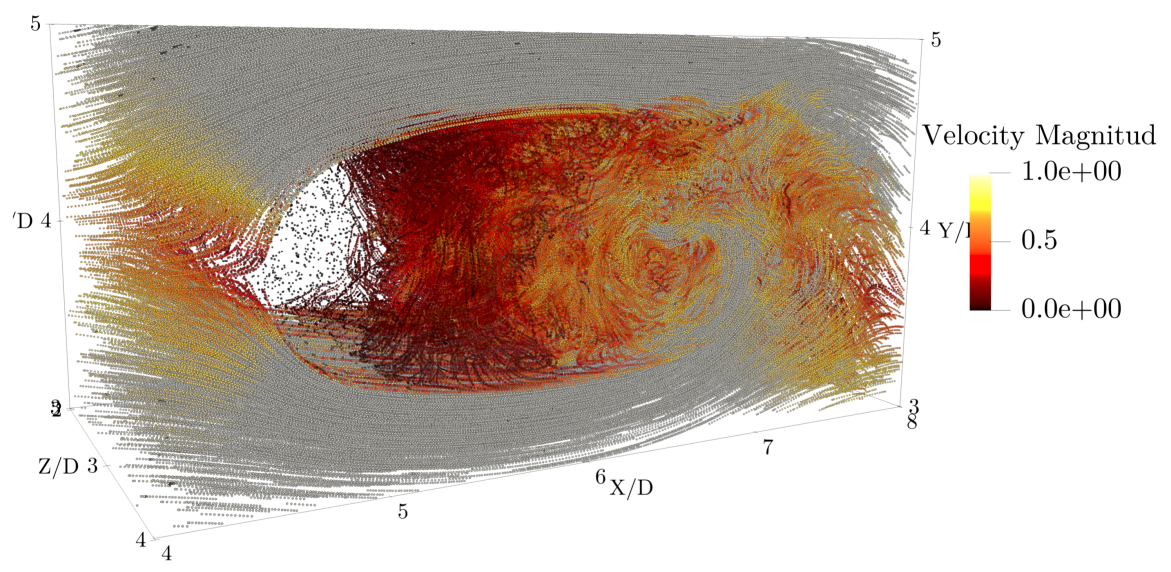

Figure 3: Lagrangian particle trajectories obtained from transport of synthetic particles through DNS Eulerian fields.

The coherent prediction function adds information obtained from temporal / local spatial Lagrangian coherent particles to come up with additional constraints in the polynomial cost function (Equation 3 ). In the worst-case scenario where there is no coherent neighbour information, the prediction function is just a simple polynomial function without additional constraints. Each particle carries sets of information, including position, first and higher-order derivative values. Assuming positions of at least three time steps $m$ are known. In this function, we impose first and second-order derivatives of each coherent particle into the prediction function as below,

$$
\begin{array}{r}
\sum_{i=2}^{n+1} a_{i, n+1} \cdot(i-1) * t_{n}^{i-2}=\ddot{y}_{n}{ }^{L C S} \\
\sum_{i=3}^{n+1} a_{i, n+1} \cdot(i-1) *(i-2) * t_{n}^{i-3}=\ddot{y}_{n}^{L C S} .
\end{array}
$$

Therefore, each particle will end up with the weighted averaged of local coherent velocity and coherent acceleration values $\left(\dot{y}_{L C S}, \ddot{y}_{L C S}\right)$. In the present study, we take four time step histories of particles to minimise the cost function and then predict the next step. The first and second derivatives of all coherent particles are weighted averaged based on their FTLE level and distance to the target particle. Two weighted averaged values, velocity and acceleration, of each target particle can be obtained for the estimation. Therefore, the modified cost function (i.e. coherent predictor) can be written as,

$$
J=\frac{1}{n} \sum_{i=1}^{n}\left(X_{i}-y_{i}\right)^{2}+\left(\dot{X}_{n}-\dot{y}_{n}\right)^{2}+\left(\ddot{X}_{n}-\ddot{y}_{n}\right)^{2} .
$$

Velocity constraint controls the direction of the prediction function, while in the case of having high acceleration gradients, a second-order constraint is required to control the acceleration of the prediction. The solution for the cost function in Equation 5 is not only smooth on the history of the target particle but also satisfies local coherent first and second-order derivatives. We compared the performance of the coherent predictor with three other prediction functions, as listed in Table 1. DNS predictor was defined as a reference using the Euler equation to transport particle positions by the ground truth DNS velocity. Predicted particle positions are followed by shaking or other optimisation techniques. Therefore, all particles are either tracked or untracked except for the inlet and outlet trajectories. In every time-step, untracked particles are like additive noises and might gradually cause to collapse of the whole trajectory process. Due to this, untracked particles must be fed by other complementary treatments. To this end, new information can be extracted if any groups of tracked particles are found to be located in the neighbourhood of untracked particles with a time step phase delay (i.e. $t_{n+1}$ ). This phase delay means that new tracked particles at time-step $t_{n+1}$ are 


\begin{tabular}{llll}
\hline Method & Fit parameters & Cost function & Prediction \\
\hline a) DNS predictor & - & & $X_{n+1}=\dot{X}_{D N S} \cdot t_{n+1}$ \\
b) Polynomial predictor & $\sum_{j=n-\ell}^{n} \sum_{i=1}^{n+1} a_{i, j} \cdot t_{j}^{i-1}=y_{n-\ell, n}$ & $J=\frac{1}{n} \sum_{i=n-m}^{n}\left(X_{i}-y_{i}\right)^{2}$ & $\mathrm{y}_{n+1}=\sum_{i=1}^{n+1} a_{i, n+1} \cdot t_{n+1}^{i-1}$ \\
c) Wiener filter & $\sum_{i=1}^{\ell} w_{i} \cdot u_{n}=y_{n}$ & $J=\left(X_{n}-u_{n}^{T} w\right)^{2}$ & $y_{n+1}=\sum_{i=2}^{\ell+1} w_{i} \cdot u_{n}$ \\
d) Coherent predictor & $\sum_{j=n-\ell}^{n} \sum_{i=1}^{n+1} a_{i, j} \cdot t_{j}^{i-1}=y_{n-\ell, n}$ & $J=\frac{1}{n} \sum_{i=1}^{n}\left(X_{i}-y_{i}\right)^{2}+\left(\dot{X}_{n}-\dot{y}_{n}\right)^{2}+\left(\ddot{X}_{n}-\ddot{y}_{n}\right)^{2}$ & $\mathrm{y}_{n+1}=\sum_{i=1}^{n+1} a_{i, n+1} \cdot t_{n+1}^{i-1}$ \\
& $\sum_{i=2}^{n+1} a_{i, n+1} \cdot(i-1) * t_{n}^{i-2}=\dot{y}_{n}^{L C S}$ & & \\
& $\sum_{i=3}^{n+1} a_{i, n+1} \cdot(i-1) *(i-2) * t_{n}^{i-3}=\ddot{y}_{n}^{L C S}$ & & \\
\hline
\end{tabular}

Table 1: Prediction function formulation

locally coherent with one specific untracked particle at time-step $t_{n}$. Another technique to reduce the number of untracked particles is to use backward prediction, which is well established in classic schemes such as nearest neighbour trajectory. Similarly, we implemented the backward predictor to search for additional information from the coherent particles to estimate in reverse pace followed by backward shaking. On the one hand, surrounding information of an untracked particle can provide the least information to predict in backward pace. In addition, this treatment can also connect spilt tracklets for reconstructing longer particle trajectories. This process is iterative, meaning that every forward step is embedded with a series of backward estimations from the current time-step up to the first step. In the present study, we evaluated and compared the performance of coherent predictor only in forward prediction.

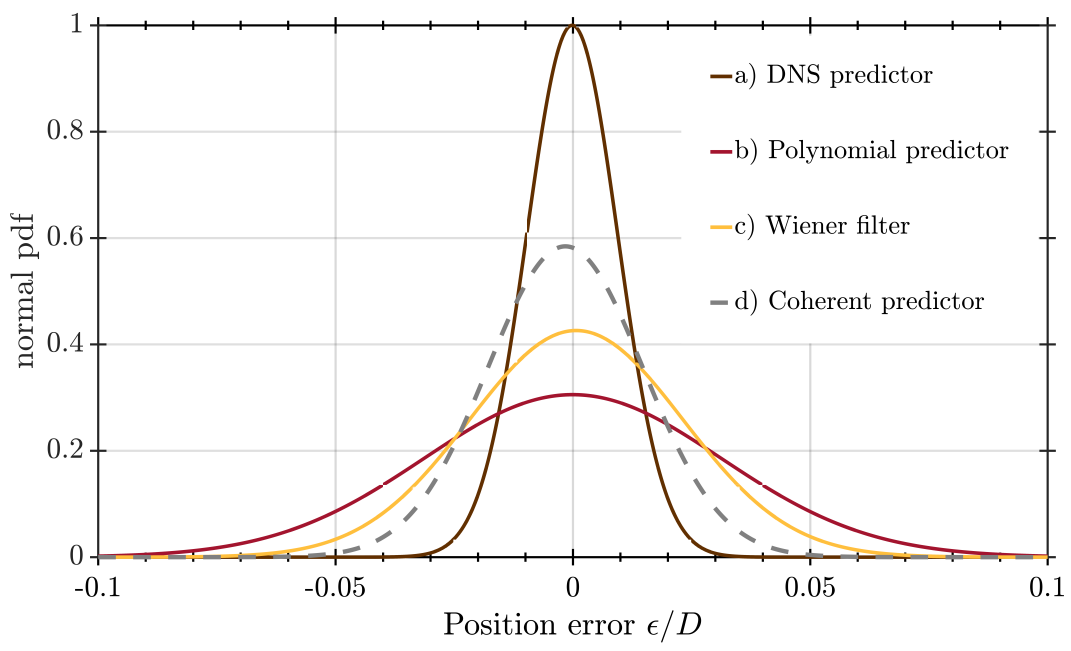

Figure 4: Normal pdf of particle position error in $\mathrm{x}$ direction of four predictor functions.

\section{Synthetic Evaluation}

To evaluate our novel particle position prediction scheme, we used a DNS simulation of the wake behind a smooth cylinder at Reynolds number equal to 3900 computed by an open-access code named Incompact $3 \mathrm{~d}$ [12]. Particle trajectories around the cylinder are shown in Figure 3. Particles are transported by every 10 DNS time step using the fourth order Runge Kutta temporal and trilinear spatial schemes. The synthetic dataset is available to the public for interested readers [13, 14]. In the synthetic case, particle trajectories are smooth and predictable when the synthetic temporal scale is with the same order of the DNS time step due to the small travelling distance between two time steps (less than the Kolmogorov timescale). However, the travelling distance is comparably large for a real PTV experiment. To mimic the real experiment, we created 

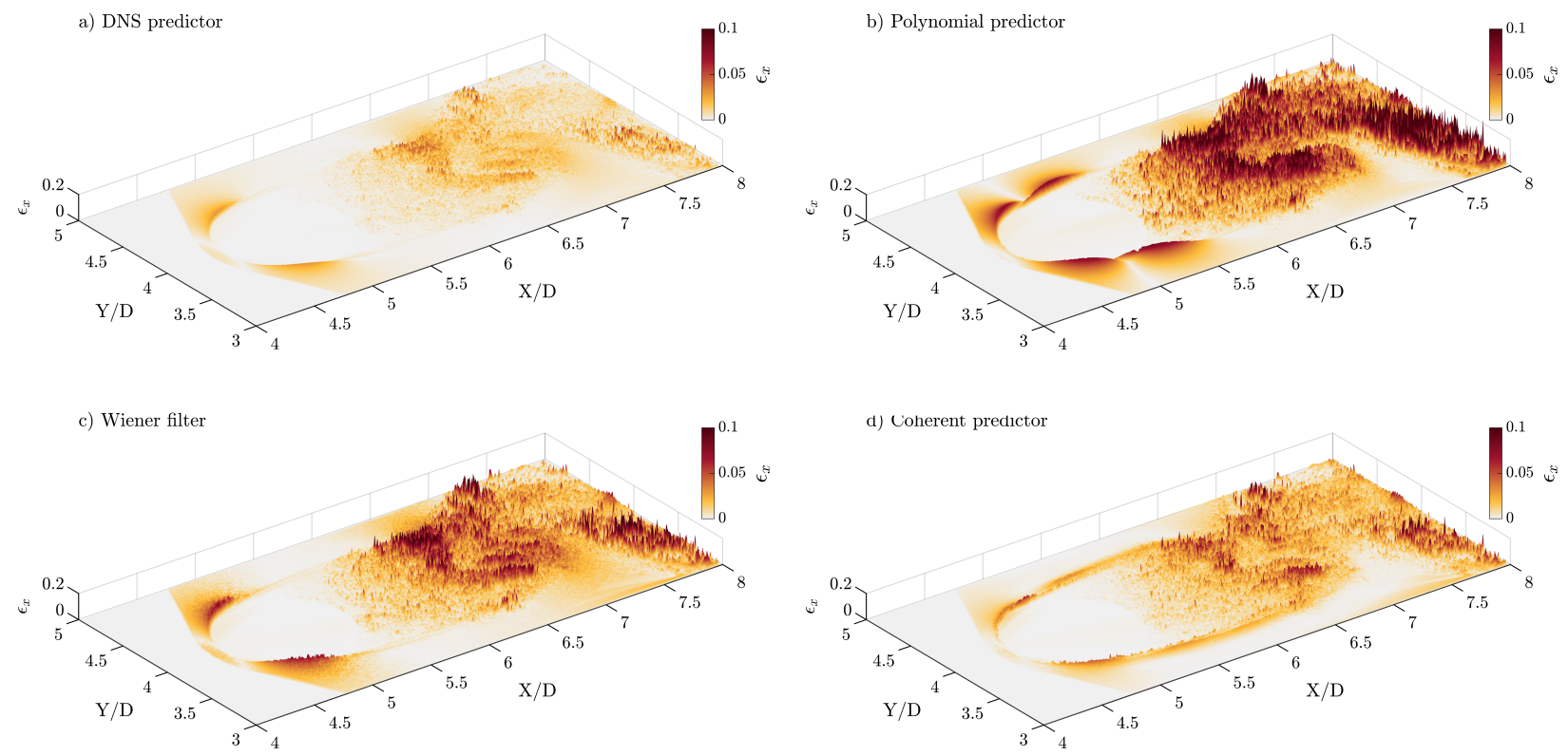

Figure 5: Position estimation error averaged in z direction: a), DNS predictor; b), Polynomial Predictor; c), Wiener filter; d), Coherent predictor.

around 150,000 ground truth trajectories associated with noise for every 20 DNS time step. By increasing the temporal scale, less particle position temporal information is available, and then the prediction would be more challenging. Therefore, even a weak signal of coherent motion would lead to a better prediction.

We compared position prediction of four schemes with the ground truth particle trajectories (see Table 1). First, we predicted particle positions based on known ground truth DNS velocity with a linear Euler transport function. In such a scenario, we can estimate the minimum uncertainty level that can be achieved in this sparse temporal scale. Both Wiener filter and Polynomial predictors are also selected to be compared with the LCS based predictor (i.e. coherent predictor). Figure 4 shows normal pdf of the predicted position error in $x$ direction of four schemes. Position error in $x$ direction shows that deviations for coherent predictor stay virtually below $0.05 \varepsilon / D$, where $\mathrm{D}$ is the cylinder diameter. On the contrary, a significant number of particles are mispredicted in both Polynomial and Wiener filter techniques. Similar significant improvements of using coherent predictor are observed in $y$ and $z$ directions. Figure 5 shows the projected distribution of the position error on $x y$ plane for each predictor function. Interestingly, the prediction error is highly correlated with the flow behaviour. Although the DNS predictor (see Figure 5.a) uses known ground truth velocity information, the travelling distance is large enough to introduce the prediction errors, particularly inside the wake region. As shown in Figure 5 b, third order polynomial has the worst prediction error, which can be up to $0.2 \varepsilon / D$ around the cylinder leading edge and inside the wake region. The polynomial prediction error distribution is fully shaped by the flow motion meaning that any gradients inside the flow create huge estimation error. Overall and local performance of the Wiener filter is better than the Polynomial predictor. Wiener filter succeeded to reduce the prediction error in most of the peak regions (see Figure 5 b.c). Error distribution reveals that coherent predictor has the best performance locally and globally compared to Wiener and Polynomial predictors. Worth mentioning that a small prediction error reduces the probability of picking a wrong particle from the surroundings in the optimisation process of 4D-PTV.

\section{Experimental Evaluation}

An experimental study of the cylinder wake flow at Reynolds number equal to 3900 (same value as the synthetic data) was performed. We designed an experimental setup with four cameras as shown in Figure 6.c. Four CMOS SpeedSense DANTEC cameras with a resolution of $1280 \times 800$ pixels and the maximum frequency of $3 \mathrm{kHz}$ are empowered. Cameras are equipped with Nikon $105 \mathrm{~mm}$ lenses. The first two cameras are positioned in backward light scattering, while the second two cameras received maximum intensity signal in forward scattering. The calibration error was lower than 0.06 pixel and reduced to 0.04 after the 
a)

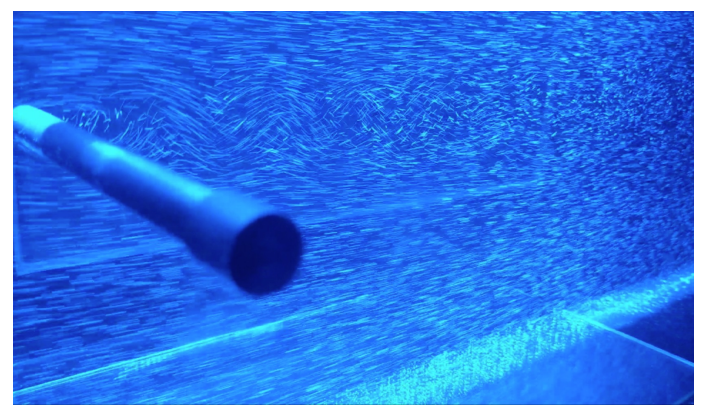

b)

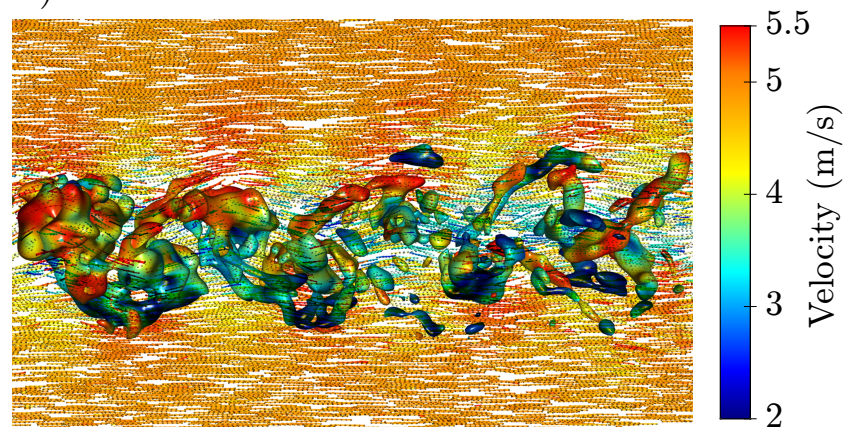

c)

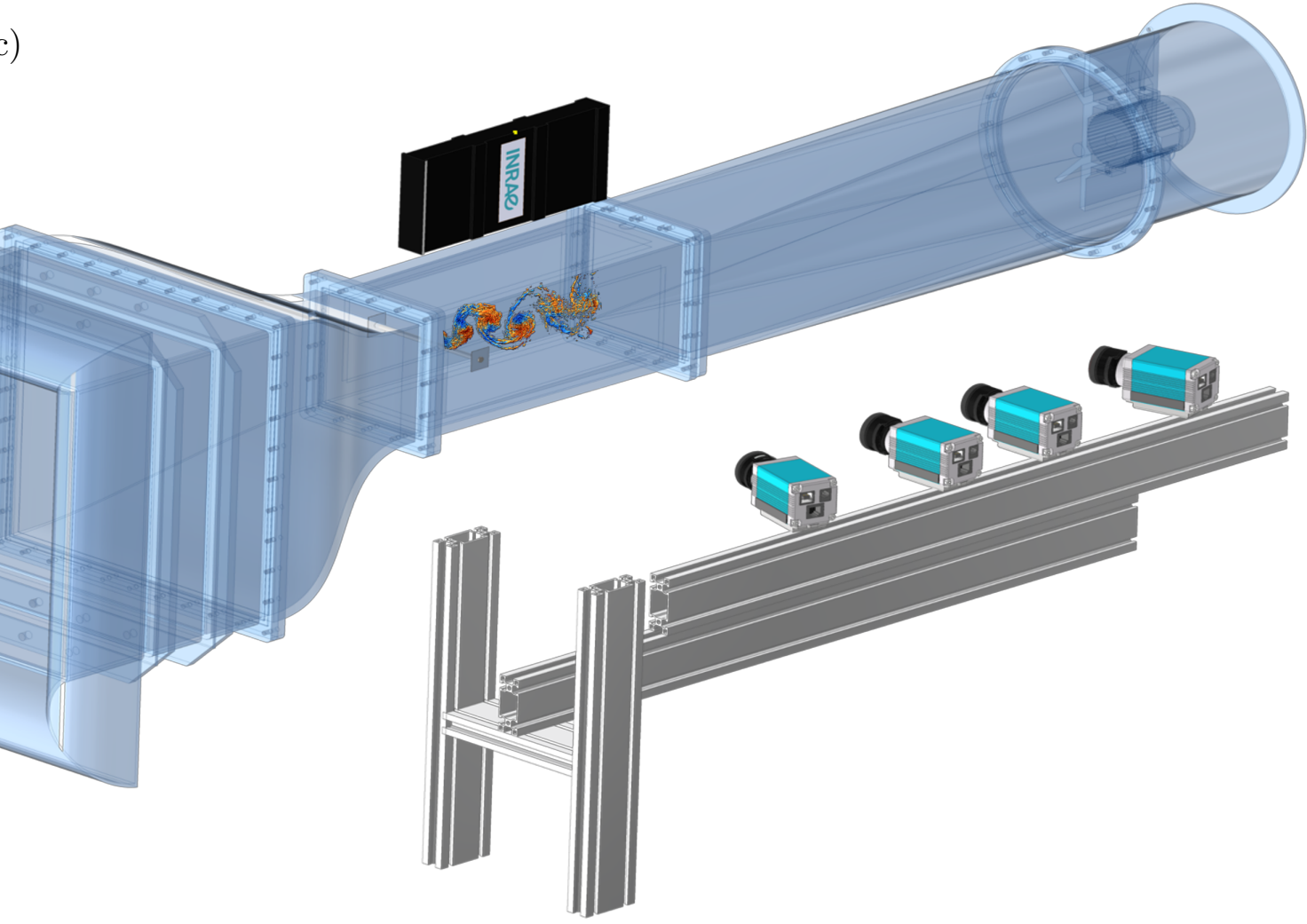

Figure 6: The cylinder wake flow at Reynolds 3900 with four cameras: a), Snapshot of the 4D-PTV experiment; b), Side view of particle trajectories superimposed by vorticity iso-surfaces; c), Schematic of the experimental setup.

volume self calibration. The volume of interest was $200 \mathrm{~mm} \times 150 \mathrm{~mm} \times 46 \mathrm{~mm}$ starting from roughly $4 D$ downstream of the cylinder, knowing that the vortex formation zone ends at $4 D$. The aperture was set at 11 to achieve $46 \mathrm{~mm}$ depth of focus. We used an LED system to illuminate this large volume. The seeding particles were Helium Filled Soap Bubbles (HFSB) [15] resulting desired intensity signal with appropriate particle size. However, bubbles are limited by three main factors in the wind tunnel experiments, including generation rate, lifetime, and image glare points. We placed 50 bubble generator nozzles with airfoil-shaped structures inside the wind tunnel chamber. The nozzles were far upstream of the measurement section to ensure a sufficient number of bubbles are created, and the main flow field is not disturbed by the existence of nozzles. The bubble lifetime is very short (less than $2-3$ minutes) inside the wind tunnel, mainly because they explode by passing through honeycomb layers. To overcome this issue, we injected bubbles inside the chamber for up to 5 minutes when the wind tunnel is off before starting the acquisition. We found that particles larger than three pixels create two glare points on two sides of the bubble. This requires more 


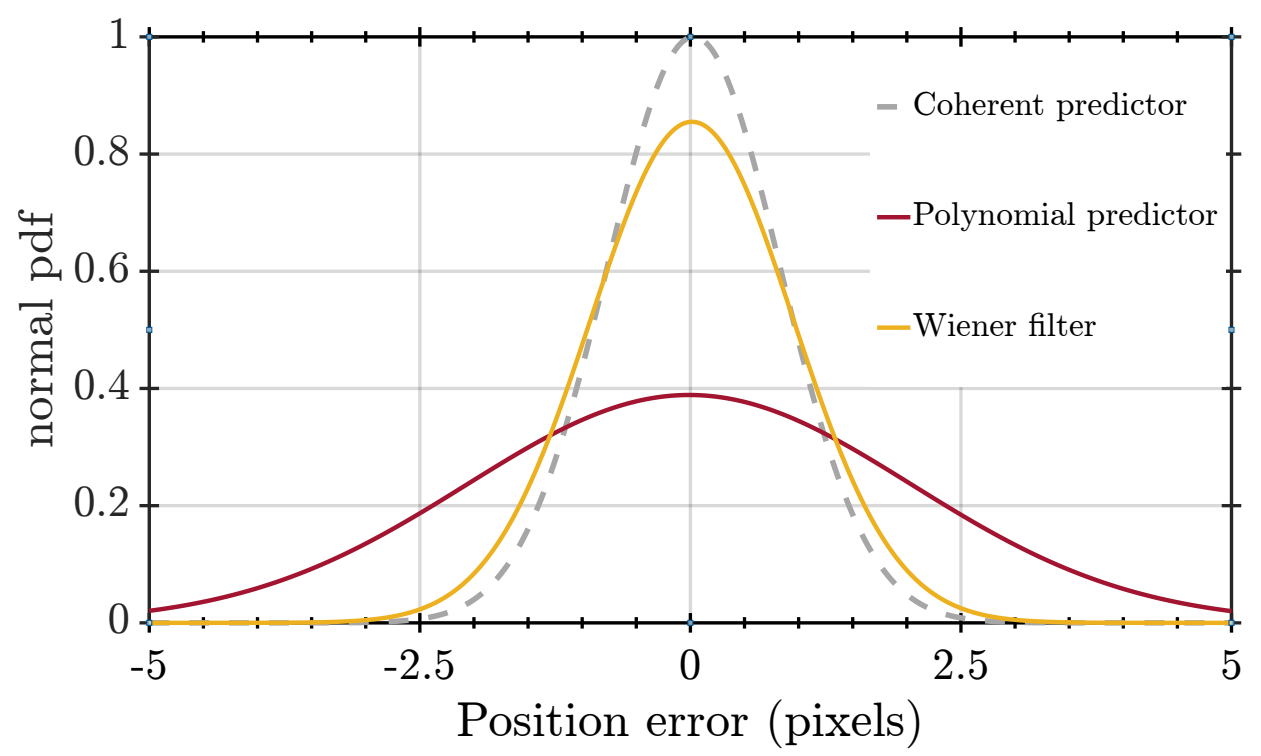

Figure 7: Experiment normal pdf results of particle position error in $\mathrm{x}$ direction of three predictors. Each predictor is compared with final optimised positions of STB Davis.

image treatments before running the 4D-PTV algorithm to avoid false particle reconstruction. However, the intensity of two glare points can diffuse and merge if the particle size is around two pixels. Therefore, we adjusted the camera magnification to reach two particle pixel sizes on average to surpass the glare point issue. One snapshot of the experiment is shown in Figure 6a. Trajectory results of the current experiment with superimposed vorticity iso-surfaces are shown in Figure 6 b.

To quantify the results of different schemes, we compared predictions with optimised positions obtained from STB Davis. As a result of the experiment, STB managed to successfully build nearly 12000 particles as shown in Figure 5.b. Noisy particle reconstruction of four time steps was used as an input of the prediction functions. We compared three techniques, Polynomial, Wiener filter, and coherent predictors, with final optimised positions. The deviation of position estimated of each technique is shown in Figure 7. The distribution shows that the coherent predictor has more accurate estimations within 1 pixel deviation from the optimised positions. Position estimations of Wiener filter and coherent predictors stay below 2.5 pixels deviation for nearly all particles. On the contrary, the Polynomial predictor has maximum deviation with STB Davis.

\section{6 conclusion}

We proposed a robust technique to predict particle positions based on their local temporal and spatial coherent motions. LCS can classify and divide the coherent neighbour motions. We imposed first and secondorder derivatives of the neighbour coherent motions into the predictor function in addition to the particle history. To assess the proposed method named coherent predictor, we performed the synthetic analysis of the wake behind a smooth cylinder at Reynolds number equal to 3900. We compared three predictor functions. Polynomial predictor showed maximum deviation with the ground truth data. Whereas coherent predictor provided the most accurate position estimation. We found that the flow regions highly impact the estimation error. Inside the wake region, particularly the vortex formation zone and the two sideward shear layers, cause more challenges in prediction. These mentioned regions are featured by high acceleration and 3D directional motions. We also performed the 4D-PTV experiment of the wake flow behind a cylinder at the same Reynolds number. It was found that the coherent predictor is reliable to estimate particle positions very close to the optimised positions. 


\section{References}

[1] Ali Rahimi Khojasteh, Yin Yang, Dominique Heitz, and Sylvain Laizet. Lagrangian Coherent Track Initialisation (LCTI). In arXiv, volume 9, 2021.

[2] Daniel Schanz, Sebastian Gesemann, and Andreas Schröder. Shake-The-Box: Lagrangian particle tracking at high particle image densities. Experiments in Fluids, 57:1-27, 52016.

[3] Yin Yang and Dominique Heitz. Kernelized Lagrangian Particle Tracking. 42021.

[4] Daniel Schanz, Andreas Schröder, Sebastian Gesemann, Dirk Michaelis, and Bernhard Wieneke. 'Shake The Box': A highly efficient and accurate Tomographic Particle Tracking Velocimetry (TOMOPTV) method using prediction of particle positions. In 10th International Symposium on Particle Image Velocimetry, 2013.

[5] Andreas Schröder, Daniel Schanz, Dirk Michaelis, Christian Cierpka, Sven Scharnowski, and Christian J. Kähler. Advances of PIV and 4D-PTV "shake-The-Box" for Turbulent Flow Analysis -the Flow over Periodic Hills. Flow, Turbulence and Combustion, 95(2-3):193-209, 2015.

[6] Daniel Schanz, Andreas Schröder, and Sebastian Gesemann. 'Shake The Box' - a 4D PTV algorithm: Accurate and ghostless reconstruction of Lagrangian tracks in densely seeded flows. 17th International Symposium on Applications of Laser Techniques to Fluid Mechanics, pages 7-10, 2014.

[7] Shiyong Tan, Ashwanth Salibindla, Ashik Ullah, and Mohammad Masuk. An open-source Shakethe-Box method and its performance evaluation. In 13th International Symposium on Particle Image Velocimetry, volume i, 2019.

[8] Andreas Schröder, Daniel Schanz, Reinhard Geisler, Sebastian Gesemann, and Christian Willert. Nearwall turbulence characterization using 4D-PTV Shake-The-Box. In 11th International Symposium on Particle Image Velocimetry, 2015.

[9] George Haller. Lagrangian coherent structures. Annual Review of Fluid Mechanics, 47:137-162, 2015.

[10] Ali Rahimi Khojasteh, Dominique Heitz, Yin Yang, and Sylvain Laizet. Lagrangian Coherent Track Initialisation. In 3rd Workshop and 1st Challenge on Data Assimilation \& CFD Processing for PIV and Lagrangian Particle Tracking, 2020.

[11] Shawn C. Shadden, Francois Lekien, and Jerrold E. Marsden. Definition and properties of Lagrangian coherent structures from finite-time Lyapunov exponents in two-dimensional aperiodic flows. Physica D: Nonlinear Phenomena, 212(3-4):271-304, 122005.

[12] Sylvain Laizet and Ning Li. Incompact3d: A powerful tool to tackle turbulence problems with up to $\mathrm{O}(105)$ computational cores. International Journal for Numerical Methods in Fluids, 67:1735-1757, 122011.

[13] Ali Rahimi Khojasteh, Sylvain Laizet, Dominique Heitz, and Yin Yang. Lagrangian and Eulerian dataset of flow over a circular cylinder at Reynolds number 3900, 2021.

[14] Ali Rahimi Khojasteh, Sylvain Laizet, Dominique Heitz, and Yin Yang. Lagrangian and Eulerian dataset of the wake over a smooth cylinder at a Reynolds number equal to 3900. Data in Brief, 2021.

[15] Fulvio Scarano, Sina Ghaemi, Giuseppe Carlo Alp Caridi, Johannes Bosbach, Uwe Dierksheide, and Andrea Sciacchitano. On the use of helium-filled soap bubbles for large-scale tomographic PIV in wind tunnel experiments. Experiments in Fluids, 56:1-12, 22015. 